05,04

\title{
Мессбауэровские исследования и микроволновые свойства гексаферритов бария с замещением ионами $\mathrm{Al}^{3+}$ и $\mathrm{In}^{3+}$
}

\author{
(C) А.В. Труханов ${ }^{1,2}$, В.Г. Костишин ${ }^{2}$, В.В. Коровушкин ${ }^{2}$, Л.В. Панина ${ }^{2}$, С.В. Труханов ${ }^{1}$, \\ В.А. Турченко , И.С. Поляков ${ }^{2}$, Р.Х. Рахматуллин ${ }^{2}$, Г.А. Филатов ${ }^{2}$, Т.И. Зубарь ${ }^{4}$, \\ В.В. Олейник ${ }^{5}$, Е.С. Яковенко ${ }^{5}$ Л.Ю. Мацуй ${ }^{5}$, Л.Л. Вовченко ${ }^{5}$, \\ В.Л. Лаунец ${ }^{5}$, Е.Л. Труханова ${ }^{1,2}$ \\ ${ }^{1}$ НПЦ НАН Беларуси по материаловедению, \\ Минск, Беларусь \\ ${ }^{2}$ Национальный исследовательский технологический университет „МИСиС“, \\ Москва, Россия \\ ${ }^{3}$ Лаборатория нейтронной фризики им. И.М. Франка, Объединенный институт ядерных исследований, \\ Дубна, Россия \\ ${ }^{4}$ Институт тепло- и массообмена им. А.В. Лыкова НАН Беларуси, \\ Минск, Беларусь \\ ${ }^{5}$ Киевский национальный университет им. Тараса Шевченко, \\ Киев, Украина \\ E-mail: truhanov86@mail.ru
}

\begin{abstract}
Представлены результаты исследования корреляции химического состава, структуры и микроволновых характеристик твердых растворов гексаферрита бария $\mathrm{BaFe}_{12-x} \mathrm{D}_{x} \mathrm{O}_{19}(0.1 \leq x \leq 1.2)$ с замещением диамагнитными ионами $\mathrm{Al}^{3+}$ и $\mathrm{In}^{3+}$. Прецизионные данные о кристаллической структуре получены методом порошковой нейтронной дифракции на Фурье-дифрактометре высокого разрешения (Дубна, ОИЯИ). Данные о распределении диамагнитных ионов-заместителей в структуре гексаферрита были получены методом мессбауэровской спектроскопии. Микроволновые свойства (коэффициента пропускания и отражения) были исследованы в диапазоне частот $20-65 \mathrm{GHz}$ и во внешних магнитных полях до $8 \mathrm{kOe.} \mathrm{Было} \mathrm{отмечено,}$ что спектры пропускания характеризуются пиком, соответствующим резонансной частоте поглощения электромагнитной энергии, что обусловлено явлением ферромагнитного резонанса. Выявлена корреляция химического состава, особенностей распределения ионов в структуре и электромагнитных свойств. Показано, что внешние магнитные поля смещают пик поглощения электромагнитного излучения в область больших частот за счет увеличения магнитокристаллической анизотропии. На основе полученных данных сделан вывод о том, что особенности внутриподрешеточных взаимодействий и анализ электромагнитных свойств должны быть объяснены с применением феноменологической модели Гуденафа-Канамори.
\end{abstract}

Работа была поддержана грантом БРФФИ Ф17Д-003.

DOI: 10.21883/FTT.2018.09.46390.093

\section{1. Введение}

Кристаллическая структура гексагональных ферритов М-типа и их твердых растворов имеет структуру магнитоплюмбита $\mathrm{PbFe}_{12} \mathrm{O}_{19}$, которая была впервые изучена Адельскольдом [1] в 1938 г. Как правило, она хорошо описана в рамках пространственной группы $P 6_{3} / m m x$ (№ 194) с гексагональной элементарной ячейкой $a=b \approx 5.90 \AA, c \approx 23.30 \AA$, содержащей две молекулы на формульную единицу. Ионы железа распределены в пяти неэквивалентных кристаллографических положениях (анионные окружения): $2 a, 4 f_{2}$ и $12 k-$ октаэдрические, $2 b$ - бипирамидальное и $4 f_{1}-$ тетраэдрическое кислородные окружения. Эти соединения характеризуются сосуществованием сильных внутриподрешеточных обменных взаимодействий Гейзенберга и слабым конкурированием межподрешеточных обменных взаимодействий [2]. Как правило, внутриподрешеточные взаимодействия доминируют и образуют коллинеарную ферримагнитную структуру с большими энергиями об- менных взаимодействий. Сильная корреляция внутриподрешеточных обменных взаимодействий и ослабление межподрешеточного обмена может привести к образованию неколлинеарных нетипичных магнитных структур (конические, геликоидальные магнитные структуры и т.д.).

$\mathrm{BaFe}_{12} \mathrm{O}_{19}$ гексагональный феррит бария или BFO и твердые растворы $\mathrm{BaFe}_{12-x} \mathrm{D}_{x} \mathrm{O}_{19}$ или BFDO на его основе (с изовалентным или гетеровалентным диамагнитным замещением $\mathrm{Fe}^{3+}$ в В-подрешетке) привлекают большое внимание исследователей [3-8] благодаря отличным функциональным свойствам [9]. Химическая инертность и коррозионная стойкость [10] делают их экологически безопасными и стабильными. Сосуществование огромной коэрцитивной силы, высокой остаточной намагниченности и высокого электросопротивления делает эти материалы пригодными для практического применения. До недавнего времени BFO и BFDO широко использовались только в качестве постоянных магнитов [11]. Даже в настоящее время более 80\% 
коммерческих постоянных магнитов в мире являются гексагональными ферритами. Также гексаферриты рассматривались как перспективные материалы для устройств магнитной записи (перпендикулярный тип намагниченности) [12]. В последнее время отмечается значительный рост публикаций по BFO и BFDO, вызванных их мультиферроидными свойствами (сосуществование ферримагнитного и сегнетоэлектрического упорядочения) [13-18]. Высокое электросопротивление данных материалов позволяет использовать их для высокочастотных применений (как элементы функциональных устройств СВЧ-оборудования и поглотителей электромагнитного излучения) [21-24].

Для развития мобильной связи, интернета и цифровых сетей передачи данных требуется увеличение скорости и объема передачи информации, которые возможны при переходе от сантиметрового диапазона длин волн в миллиметровый диапазон, соответствующий частотам 30-100 GHz. Гексаферриты М-типа имеют высокие значения поля магнитной кристаллографической анизотропии и способны работать в этом диапазоне. Данные материалы перспективны для разработки и создания функциональных устройств СВЧ-электроники: вентили, циркуляторы, фазовращатели, элементы антенн приемопередачи, а также эффективных поглотителей ЭМИ для обеспечения электромагнитной совместимости [25-30]. Допирование или замещение гексаферрита бария различными диамагнитными ионами приводит к значительному изменению магнитных характеристик и, как следствие, к изменению рабочего диапазона частот.

Соединения на основе BFO являются перспективными для поглощения (ослабления) электромагнитного излучения (ЭМИ) с учетом их магнитных свойств и возможностей их модификации. Для улучшения магнитных и функциональных свойств $\mathrm{BaFe}_{12} \mathrm{O}_{19}$ обычно используют замещение ионов железа изовалентными диамагнитными ионами $\mathrm{D}^{3+}$ (изовалентное замещение) [31-34] или гетеровалентными диамагнитными ионами $\mathrm{D}^{4+}$ или $\mathrm{D}^{2+}$ (гетеровалентное замещение). Диамагнитное замещение в BFO изменяет особенности кристаллической структуры (искажения элементарной ячейки, микродеформации и изменения длин связей $\mathrm{Fe}-\mathrm{O})$ и магнитную структуру (ослабление магнитного обменного взаимодействия и фрустрации магнитной структуры). Модификация кристаллических и магнитных структур должна изменять значение магнитокристаллической анизотропии и в результате изменять электромагнитные характеристики. Хорошо известно, что селективное поглощение ЭМИ в сложных магнитных оксидах реализуется двумя основными механизмами: резонанс доменных границ и естественный ферромагнитный резонанс - ЕФМР (частота прецессии вектора намагниченности) [35,36]. Основной вклад в поглощение на высоких частотах связан с именно с ЕФМР.

Рабочий диапазон для ВFО определяется диапазоном частот, в котором значение магнитной проницаемости (мнимой части) эффективно изменяется, что происходит вблизи ЕФМР. Использование гексагональных ферритов с высокой степенью кристаллической и магнитной анизотропии позволяет частотно-селективно управлять характеристиками ЭМИ путем смещения резонансной частоты ЕФМР. Частота ЕФМР незамещенного $\mathrm{BaFe}_{12} \mathrm{O}_{19}$ находится в области $49-50 \mathrm{GHz}$ и зависит от значения внутреннего магнитного поля анизотропии $H_{a}$ :

$$
\omega=\gamma H_{a}
$$

$\omega$ - частота ЕФМР, $\gamma$ - гиромагнитное отношение.

Область ЕФМР в поликристаллических ферритах является достаточно широкой и ее можно контролировать, изменяя значение $H_{a}$, например, путем введения в структуру феррита различных диамагнитных ионов, а также при наличии внешнего магнитного поля $H_{0}$ :

$$
f=\gamma\left(H_{0}+H_{a}-4 \pi M_{s}\right)
$$

$4 \pi M_{s}$ - размагничивающий фактор.

Таким образом, контролируя уровень ионов-заместителей, мы можем изменить амплитудно-частотные характеристики резонанса.

В данной работе мы исследовали корреляцию между концентрацией диамагнитных ионов $\left(\mathrm{Al}^{3+}, \mathrm{In}^{3+}\right)$ и электромагнитными свойствами твердых растворов гексаферрита бария $\mathrm{BaFe}_{12-x} \mathrm{D}_{x} \mathrm{O}_{19}\left(\mathrm{D}=\mathrm{Al}^{3+}\right.$ и $\mathrm{In}^{3+}$; $0 \leq x \leq 1.2)$. Диамагнитные ионы $\left(\mathrm{Al}^{3+}\right.$ и $\left.\mathrm{In}^{3+}\right)$ были выбраны по двум причинам: 1) Различие в ионных радиусах $\left(\mathrm{r}_{\mathrm{Al}}^{3+}-0.54 \AA \ll \mathrm{r}_{\mathrm{Fe}}^{3+}-0.64 \AA<\mathrm{r}_{\mathrm{In}}^{3+}-0.94 \AA\right)$; 2) Различная электронная структура ионов. Ион алюминия имеет полностью заполненную $p$-оболочку и пустую $d$-оболочку и может называться , $d^{0}$-ион“. Электронная структура $\mathrm{Al}^{3+}-2 s^{2} 2 p^{6}$ соответствует конфигурации благородного газа $\mathrm{Ne}$. Ион индия имеет полностью заполненную $d$-оболочку и пустую $s$-оболочку и может называться „, $d^{10}$-ион“ или „полностью заполненный ион“. Электронная структура $\operatorname{In}^{3+}-4 d^{10} 5 s^{0}$ соответствует электронной конфигурации благородного металла $\mathrm{Pd}$. Основная цель работы - проведение исследований корреляции химического состава, структуры и микроволновых характеристик твердых растворов гексаферрита бария $\mathrm{BaFe}_{12-x} \mathrm{D}_{x} \mathrm{O}_{19}(0.1 \leq x \leq 1.2)$ с замещением диамагнитными ионами $\mathrm{Al}^{3+}$ и $\mathrm{In}^{3+}$.

\section{2. Методика эксперимента}

Исследуемые керамические образцы $\mathrm{BaFe}_{12-x} \mathrm{D}_{x} \mathrm{O}_{19}$ $\left(\mathrm{D}=\mathrm{Al}^{3+}\right.$ и $\left.\mathrm{In}^{3+}, 0.1 \leq x \leq 1.2\right)$ были получены из оксидов $\mathrm{Fe}_{2} \mathrm{O}_{3}$ и $\mathrm{D}_{2} \mathrm{O}_{3}\left(\mathrm{D}=\mathrm{Al}^{3+}\right.$ и $\left.\mathrm{In}^{3+}\right)$ и карбоната $\mathrm{BaCO}_{3}$ c использованием двухступенчатой реакции (обычный метод твердой реакции). Все исходные реагенты были квалификации ОСЧ. Оксиды и карбонат были смешаны в строго стехиометрическом соотношении. Предварительный синтез был выполнен при $1200^{\circ} \mathrm{C}$ на воздухе в течение $6 \mathrm{~h}$. Окончательный синтез проводили при $1300^{\circ} \mathrm{C}$ на воздухе в течение $6 \mathrm{~h}$. После синтеза образец 


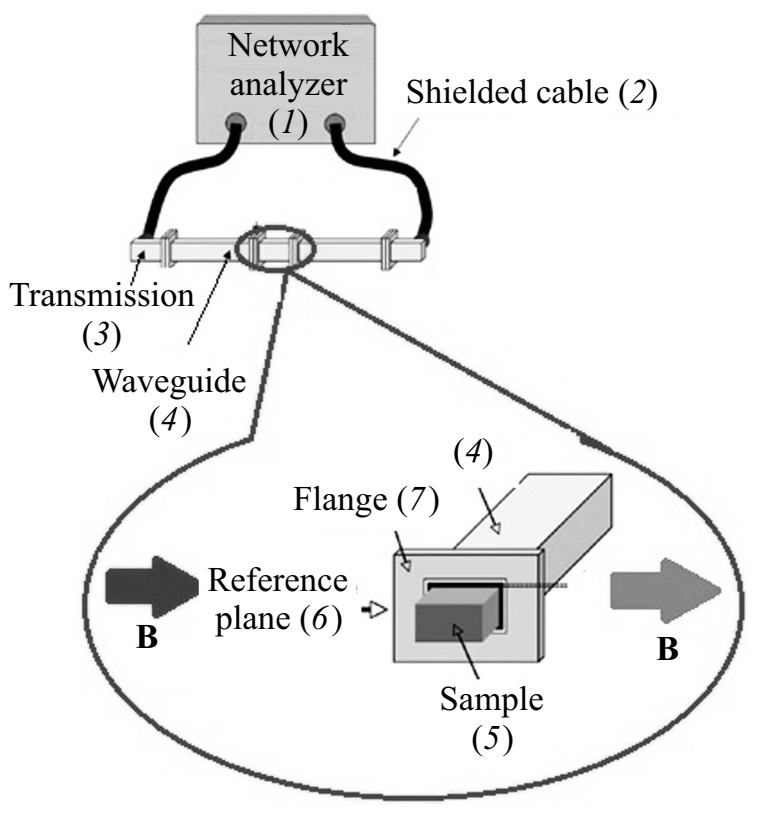

Рис. 1. Схематическое изображение метода измерения электромагнитных характеристик волноводым методом (1 - векторный анализатор цепей; 2 - экранированный кабель; 3 - выход для измерения спектров прохождения; 4 - волновод; 5 - исследуемый образец; 6 - эталонная плоскость; 7 - фланец).

медленно охлаждался $\left(100^{\circ} \mathrm{C} \cdot \mathrm{h}^{-1}\right)$ [37]. Синтез образцов $\mathrm{BaFe}_{12-x} \mathrm{D}_{x} \mathrm{O}_{19}$ может быть представлен следующей реакцией:

$$
\begin{aligned}
\mathrm{BaCO}_{3}+\left(6-\frac{1}{2} x\right) & \mathrm{Fe}_{2} \mathrm{O}_{3}+\left(\frac{1}{2} x\right) \mathrm{D}_{2} \mathrm{O}_{3} \\
& \rightarrow \mathrm{BaFe}_{12-x} \mathrm{D}_{x} \mathrm{O}_{19}+\mathrm{CO}_{2} \uparrow .
\end{aligned}
$$

Параметры элементарной ячейки $(a, c$ и $V)$ были рассчитаны на основе экспериментальных данных дифракции нейтронов. Нейтронографические исследования были проведены на Фурье-дифрактометре высокого разрешения (ФДВР). ФДВР представляет собой времяпролетный дифрактометр на импульсном реакторе ИБР-2М в Дубне с относительно большой $(\sim 21.131 \mathrm{~m})$ пролетной базой от замедлителя до детектора и обладает исключительно высокой разрешающей способность $(\delta d / d \approx 0.001)$, которая к тому же практически не зависит от межплоскостного расстояния в широком интервале $d_{h k l}$. Нейтронограммы высокого разрешения измерялись детекторами, расположенными при средних углах рассеяния $\pm 152^{\circ}$, в интервале межплоскостных расстояния от 0.6 до $3.6 \AA$. Расчет экспериментальных времяпролетных нейтронограмм выполняли методом полнопрофильного анализа Ритвельда [38] с помощью программных пакетов MRIA и FullProf [39], с использованием встроенных таблиц для длин когерентного рассеяния и магнитных формфакторов. Исследования характера распределения ионов-заместителей в структуре гексаферрита были проведены методом мессбауэровской спектроскопии на спектрометре Ms1104-Em. Обработка данных осуществлялась с помощью программного обеспечения UnivemMs. Измерения электромагнитных свойств проводились методом волноводов (рис. 1) с использованием анализатора сети Agilent.

Исследования проводились в диапазоне $20-65 \mathrm{GHz}$. Коэффициенты прохождения (передачи) $k_{\text {tr }}$ и отражения $k_{\text {ref }}$ определялись как

$$
k_{\mathrm{tr}}=10 \cdot \lg \left(\frac{P_{\mathrm{tr}}}{P_{\mathrm{inc}}}\right) \quad \text { и } \quad k_{\mathrm{ref}}=10 \cdot \lg \left(\frac{P_{\mathrm{ref}}}{P_{\text {inc }}}\right),
$$

$P_{\text {inc }}-$ исходная (падающая) мощность ЭМИ, $P_{\mathrm{tr}}$ прошедшая через образец мощность ЭМИ, $P_{\text {ref }}-$ отраженная мощность ЭМИ. Коэффициент поглощения ЭМИ $k_{\text {abs }}$ может быть рассчитан по экспериментально полученным коэффициентам $k_{\text {tr }}$ и $k_{\text {ref }}$ с учетом закона сохранения энергии по формуле

$$
k_{\mathrm{abs}}=10 \cdot \lg \left(1-10^{0.1 \cdot k_{\mathrm{tr}}}-10^{0.1 \cdot k_{\mathrm{ref}}}\right) .
$$

\section{3. Экспериментальные результаты и их обсуждение}

Согласно данным нейтронной дифракции, все исследованные образцы $\mathrm{BaFe}_{12-x} \mathrm{D}_{x} \mathrm{O}_{19} \quad\left(\mathrm{D}=\mathrm{Al}^{3+}\right.$ и $\mathrm{In}^{3+}$, $0.1 \leq x \leq 1.2)$ характеризуются гексагональной структурой с пространственной группой $\left(P 6_{3} / m m c\right)$ с двумя молекулами в элементарной ячейке $(Z=2)-$ рис. 2 . Было установлено, что все образцы являются однофазными. Факторы соответствия, полученные в результате обработки спектров нейтронной дифракции $\left(\mathrm{R}_{\mathrm{wp}}, \mathrm{R}_{\mathrm{exp}}\right.$, $\mathrm{R}_{\mathrm{B}}, \mathrm{R}_{\mathrm{Mag}}$ и $\left.\chi^{2}\right)$, предполагают, что исследуемые образцы характеризуются высоким качеством, а уровень обработки экспериментальных данных является релевантным. На рис. 2 показана модель структурной формулы (правый рисунок) и концентрационные зависимости параметров элементарной ячейки $(a, c$ и $V)$ для $\mathrm{BaFe}_{12-x} \mathrm{Al}_{x} \mathrm{O}_{19}$ (рис. 2, $a$ ), $\mathrm{BaFe}_{12-x} \mathrm{In}_{x} \mathrm{O}_{19}$ (рис. 2, $b$ ).

Объем элементарной ячейки $\mathrm{Al}$-замещенных гексаферритов меньше, чем для незамещенного $\mathrm{BaFe}_{12} \mathrm{O}_{19}$. В то время как концентрация алюминия увеличивалась с 0.1 до 1.2 , параметры ячейки и объем элементарной ячейки уменьшались (рис. 2,a). Монотонное уменьшение объема от $696.91 \AA^{3}$ до $690.12 \AA^{3}$ объясняется незначительным уменьшением параметров $a$ (от 5.889 до $5.870 \AA$ ) и $c$ (с 23.172 до $23.126 \AA)$ в диапазоне $0.1 \leq x \leq 1.2$. Причина этой зависимости заключается в меньшем ионном радиусе ионов $\mathrm{Al}^{3+}(0.540 \AA)$ по сравнению с ионным радиусом $\mathrm{Fe}^{3+}(0.640 \AA)$. В случае In-замещенных образцов ситуация противоположная. В то время как концентрация $\mathrm{In}^{3+}$ увеличивается, параметры элементарной ячейки также увеличивались (рис. $2, b$ ). Почти линейное увеличение объема от $698.82 \AA^{3}$ до $714.63 \AA^{3}$ обусловлено значительным ростом параметров $a$ (от 5.85 до $5.932 \AA)$ и $c$ (с 23.217 до $23.445 \AA$ ) в диапазоне 


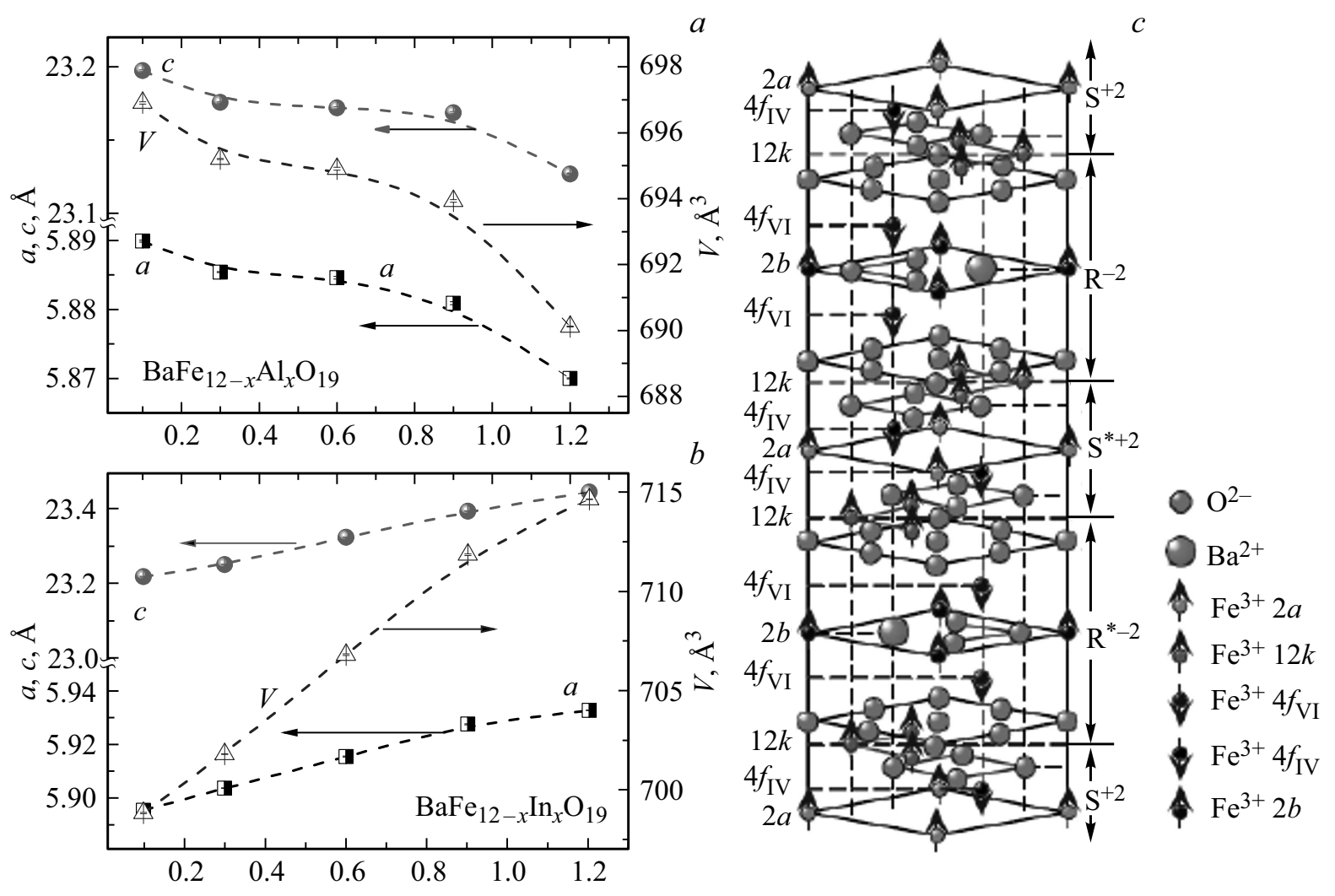

Рис. 2. Модель структурной формулы гексаферрита бария (правый рис.) и концентрационные зависимости параметров элементарной ячейки $\left(a, c\right.$ и $V$ ) для $\mathrm{BaFe}_{12-x} \mathrm{Al}_{x} \mathrm{O}_{19}$ (рис. 2, $\left.a\right), \mathrm{BaFe}_{12-x} \operatorname{In}_{x} \mathrm{O}_{19}$ (рис. 2, $b$ ).

$0.1 \leq x \leq 1.2$. Можно утверждать, что это является результатом замещения ионов железа ионом с большим ионным радиусом - $\operatorname{In}^{3+}(0.940 \AA)$.

Для определения характера распределения ионовзаместителей в структуре гексаферрита и анализе влияния занятых диамагнитными ионами позиций на интенсивность обменных взаимодействий мы применили мессбауэровскую спектроскопию. На рис. 3 представлены мессбауэровские спектры для $\mathrm{BaFe}_{12-x} \mathrm{Al}_{x} \mathrm{O}_{19}$ (рис. 3, a) и $\mathrm{BaFe}_{12-x} \operatorname{In}_{x} \mathrm{O}_{19}$ (рис. 3,b), где $x=0.1$ и 1.2. Особенности параметров Мессбауэра приведены в таблице. Незамещенный $\mathrm{BFO}(x=0)$ имеет 5 секстетов в соответствии с позициями $\mathrm{Fe}^{3+}$ (координации кислорода): октаэдрические $12 k, 4 f_{2}, 2 a$, тетраэдрические $4 f_{1}$ и бипирамидальный $2 b$. Магнитные поля в ядрах $\mathrm{Fe}^{57}$ для всех положений различаются и имеет следующий порядок: $\mathrm{B}_{\text {eff }} \mathrm{Fe}\left(4 f_{2}\right)>\mathrm{B}_{\text {eff }} \mathrm{Fe}(2 a)>\mathrm{B}_{\text {eff }} \mathrm{Fe}\left(4 f_{1}\right)>$ $\mathrm{B}_{\text {eff }} \mathrm{Fe}(12 k)>\mathrm{B}_{\text {eff }} \mathrm{Fe}(2 b)$.

Для всех образцов BFDO с $x=0,1$ наблюдалось появление нового 6-го секстета. Появление дополнительных новых секстетов, обозначенных как $12 k^{1}$, соответствует искаженному типу подрешетки $12 k$. Мы предполагаем, что диамагнитные катионы при малых концентрациях распределяются в $2 b$-позиции, что приводит к ослаблению обменных связей $\mathrm{Fe}^{3+}(2 b)-\mathrm{O}-\mathrm{Fe}^{3+}(12 k)$ и $\mathrm{Fe}^{3+}(2 b)-\mathrm{O}-\mathrm{Fe}^{3+}(2 a)$ (за счет фрустрации магнитной структуры при ослаблении дальнего порядка обменных взаимодействий). В результате этого появляются неэквивалентные положения $\mathrm{Fe}^{3+}$ (для $12 k$-позиций) с более низким полем $\left(\mathrm{B}_{\mathrm{eff}}\right)$ в ядрах $\mathrm{Fe}^{57}$. Это соответствует секстету С6 в спектрах для $\mathrm{Fe}^{3+}\left(12 k_{1}\right)$.

Теоретически формирование состояния $12 k^{1}$ могло бы быть возможным в результате распределения катионов $\mathrm{D}^{3+}$ в $12 k$-положении, поскольку он строит триады, но общая площадь секстета для С1 $(12 k)$ и C6 $\left(12 k^{1}\right)$ не подтверждает это предположение. В то же время площадь секстета, соответствующего позиции $4 f_{1}$, увеличивается. Увеличение площади этого секстета может быть результатом суперпозиции с секстетом $2 a$. Распределение катионов в $2 b$-позиции, как было отмечено выше, приводит к ослаблению обменных взаимодействий внутри связи $\mathrm{Fe}^{3+}(2 b)-\mathrm{O}-\mathrm{Fe}^{3+}(2 a)$, что приводит к неэквивалентному состоянию $\mathrm{Fe}^{3+}$ для $2 a$-положения с более низким эффективным полем на ядрах $\mathrm{Fe}^{57}$ и с параметрами, аналогичными положению $4 f_{1}$. Частичное совпадение перекрывающихся секстетов подтверждает увеличение ширины линии $\mathrm{Fe}^{3+}$ $\left(4 f_{1}\right)$. Значения поля $\left(\mathrm{B}_{\mathrm{eff}}\right)$ на ядрах $\mathrm{Fe}^{57}$ в образцах с малым уровнем замещения $(x=0.1)$ аналогичны значениям в незамещенном гексаферрите при добавлении одного нового значения $\mathrm{B}_{\mathrm{eff}}$ для $12 k^{1}$. Таким образом, поле в ядрах $\mathrm{Fe}^{57}$ при $x=0.1$ имеет следу- 
Параметры мессбауэровской спектроскопии для исследуемых образцов $\mathrm{BaFe}_{12-x} \mathrm{Al}_{x} \mathrm{O}_{19}$ и $\mathrm{BaFe}_{12-x} \operatorname{In}_{x} \mathrm{O}_{19}(x=0.1$ и $x=1.2)$

\begin{tabular}{|c|c|c|c|c|c|}
\hline Образец & $\begin{array}{c}\text { Компонента } \\
\text { спектра }\end{array}$ & $\begin{array}{l}\text { Изомерный } \\
\text { сдвиг } \delta, \mathrm{mm} / \mathrm{s}\end{array}$ & $\begin{array}{c}\text { Квадрупольное } \\
\text { расщепление } \Delta, \mathrm{mm} / \mathrm{s}\end{array}$ & $\begin{array}{c}\text { Магнитные } \\
\text { поля } B, \mathrm{~T}\end{array}$ & $\begin{array}{c}\text { Компоненты } \\
\text { площади } S, \%\end{array}$ \\
\hline \multicolumn{6}{|c|}{ Незамещенный гексаферрит бария } \\
\hline \multirow{5}{*}{$\mathrm{BaFe}_{12} \mathrm{O}_{19}$} & $\mathrm{C} 1-12 k\left(\mathrm{Fe}^{3+}\right)_{\mathrm{VI}}$ & 0.36 & 0.41 & 41.2 & 50.4 \\
\hline & $\mathrm{C} 2-4 f_{2}\left(\mathrm{Fe}^{3+}\right)_{\mathrm{VI}}$ & 0.37 & 0.17 & 51.6 & 19.2 \\
\hline & $\mathrm{C} 3-4 f_{1}\left(\mathrm{Fe}^{3+}\right)_{\mathrm{IV}}$ & 0.27 & 0.2 & 49.0 & 18.3 \\
\hline & $\mathrm{C} 4-2 s\left(\mathrm{Fe}^{3+}\right)_{\mathrm{VI}}$ & 0.33 & 0 & 50.5 & 7 \\
\hline & $\mathrm{C} 5-2 b\left(\mathrm{Fe}^{3+}\right) \mathrm{v}$ & 0.29 & 2.21 & 40.1 & 5.1 \\
\hline \multicolumn{6}{|c|}{ Al-замещенный гексаферрит бария } \\
\hline \multirow{6}{*}{$\begin{array}{c}\mathrm{BaFe}_{11.9} \mathrm{Al}_{0.1} \mathrm{O}_{19} \\
x=0.1\end{array}$} & $\mathrm{C} 1-12 k\left(\mathrm{Fe}^{3+}\right)_{\mathrm{VI}}$ & 0.35 & 0.41 & 41.3 & 45.9 \\
\hline & $\mathrm{C} 2-4 f_{2}\left(\mathrm{Fe}^{3+}\right)_{\mathrm{VI}}$ & 0.38 & 0.18 & 51.4 & 15.8 \\
\hline & $\mathrm{C} 3-4 f_{1}\left(\mathrm{Fe}^{3+}\right)_{\mathrm{IV}}$ & 0.27 & 0.21 & 48.9 & 23 \\
\hline & $\mathrm{C} 4-2 a\left(\mathrm{Fe}^{3+}\right)_{\mathrm{VI}}$ & 0.35 & 0.03 & 50.6 & 5.5 \\
\hline & $\mathrm{C} 5-2 b\left(\mathrm{Fe}^{3+}\right)_{\mathrm{IV}}$ & 0.29 & 2.16 & 40.0 & 5.9 \\
\hline & $\mathrm{C} 6-12 \mathrm{k}\left(\mathrm{Fe}^{3+}\right) \mathrm{v}$ & 0.29 & 0.36 & 39.8 & 3.9 \\
\hline \multirow{7}{*}{$\begin{array}{c}\mathrm{BaFe}_{10.8} \mathrm{Al}_{1.2} \mathrm{O}_{19} \\
x=1.2\end{array}$} & $\mathrm{C} 1-12 k\left(\mathrm{Fe}^{3+}\right)_{\mathrm{VI}}$ & 0.34 & 0.47 & 41.5 & 16.5 \\
\hline & $\mathrm{C} 2-4 f_{2}\left(\mathrm{Fe}^{3+}\right)_{\mathrm{VI}}$ & 0.39 & 0.16 & 50.3 & 19.3 \\
\hline & $\mathrm{C} 3-4 f_{1}\left(\mathrm{Fe}^{3+}\right)_{\mathrm{IV}}$ & 0.27 & 0.26 & 46.6 & 20.3 \\
\hline & $\mathrm{C} 4-2 a\left(\mathrm{Fe}^{3+}\right)_{\mathrm{VI}}$ & 0.33 & -0.32 & 50.6 & 6.3 \\
\hline & $\mathrm{C} 5-2 b\left(\mathrm{Fe}^{3+}\right)_{\mathrm{IV}}$ & 0.26 & 2.15 & 38.3 & 6 \\
\hline & $\mathrm{C6}-12 \mathrm{k}^{\mathbf{1}}\left(\mathrm{Fe}^{3+}\right)_{\mathrm{v}}$ & 0.34 & 0.42 & 40.0 & 27.6 \\
\hline & $\mathrm{C7}-12 \mathrm{k}^{\prime \prime}\left(\mathrm{Fe}^{3+}\right)_{\mathrm{VI}}$ & 0.32 & 0.43 & 36.7 & 4 \\
\hline \multicolumn{6}{|c|}{ In-замещенный гексаферрит бария } \\
\hline \multirow{6}{*}{$\begin{array}{c}\mathrm{BaFe}_{11.9} \mathrm{In}_{0.1} \mathrm{O}_{19} \\
x=0.1\end{array}$} & $\mathrm{C} 1-12 k\left(\mathrm{Fe}^{3+}\right)_{\mathrm{VI}}$ & 0.35 & 0.41 & 41.1 & 46.0 \\
\hline & $\mathrm{C} 2-4 f_{2}\left(\mathrm{Fe}^{3+}\right)_{\mathrm{VI}}$ & 0.38 & 0.18 & 51.3 & 16.3 \\
\hline & $\mathrm{C} 3-4 f_{1}\left(\mathrm{Fe}^{3+}\right)_{\mathrm{IV}}$ & 0.27 & 0.19 & 48.7 & 22.4 \\
\hline & $\mathrm{C} 4-2 a\left(\mathrm{Fe}^{3+}\right)_{\mathrm{VI}}$ & 0.35 & 0.03 & 50.4 & 5.0 \\
\hline & $\mathrm{C} 5-2 b\left(\mathrm{Fe}^{3+}\right)_{\mathrm{IV}}$ & 0.26 & 2.16 & 40.1 & 4.6 \\
\hline & $\mathrm{C6}-12 \mathrm{k}^{\prime}\left(\mathrm{Fe}^{3+}\right)_{\mathrm{v}}$ & 0.38 & 0.51 & 34.5 & 5.7 \\
\hline \multirow{7}{*}{$\begin{array}{c}\mathrm{BaFe}_{10.8} \mathrm{In}_{1.2} \mathrm{O}_{19} \\
x=1.2\end{array}$} & $\mathrm{C} 1-12 k\left(\mathrm{Fe}^{3+}\right)_{\mathrm{VI}}$ & 0.34 & 0.38 & 39.1 & 16.1 \\
\hline & $\mathrm{C} 2-4 f_{2}\left(\mathrm{Fe}^{3+}\right)_{\mathrm{VI}}$ & 0.40 & 0.11 & 48.3 & 14.6 \\
\hline & $\mathrm{C} 3-4 f_{1}\left(\mathrm{Fe}^{3+}\right)_{\mathrm{IV}}$ & 0.30 & 0.10 & 45.9 & 28.8 \\
\hline & $\mathrm{C} 4-2 a\left(\mathrm{Fe}^{3+}\right)_{\mathrm{VI}}$ & 0.35 & 0.32 & 40.4 & 9.4 \\
\hline & $\mathrm{C} 5-2 b\left(\mathrm{Fe}^{3+}\right)_{\mathrm{IV}}$ & 0.28 & 2.31 & 36.0 & 3.7 \\
\hline & $\mathrm{C6}-12 \mathrm{k}^{1}\left(\mathrm{Fe}^{3+}\right)_{\mathrm{v}}$ & 0.34 & 0.41 & 33.5 & 20.6 \\
\hline & $\mathbf{C 7}-12 \mathbf{k}^{\prime \prime}\left(\mathrm{Fe}^{3+}\right)_{\mathrm{VI}}$ & 0.41 & -0.48 & 16.4 & 6.8 \\
\hline
\end{tabular}

ющий порядок: $\mathrm{B}_{\text {eff }} \mathrm{Fe}\left(4 f_{2}\right)>\mathrm{B}_{\text {eff }} \mathrm{Fe}(2 a)>\mathrm{B}_{\text {eff }} \mathrm{Fe}\left(4 f_{1}\right)>$ $>\mathrm{B}_{\text {eff }} \mathrm{Fe}(12 k)>\mathrm{B}_{\text {eff }} \mathrm{Fe}(2 b)>\mathrm{B}_{\text {eff }} \mathrm{Fe}\left(12 k^{1}\right)$.

Но для концентрации $x=1.2$ мы наблюдали совсем другую картину. Для всех замещенных образцов BFO c $x=1.2$ наблюдалось появление нового 7-го секстета. Мы предположили, что этот 7-й секстет можно отнести к формированию нового неэквивалентного положения $12 k^{2}$ (также искаженной неэквивалентной подрешетки
$12 k$ с разной энергией связи). Это достаточно сложный случай для однозначной интерпретации распределения катионов, но, скорее всего можно предложить следующее объяснение. Для А1-замещенных образцов $(x=1.2)$ мы считаем, что диамагнитные ионы предпочтительно занимают $2 b$ - и $12 k$-положения, что приводит к ослаблению и даже разрыву обменных связей $\mathrm{Fe}^{3+}(2 b)-\mathrm{O}-\mathrm{Fe}^{3+}(12 k)$ и $\mathrm{Fe}^{3+}(2 a)-\mathrm{O}-\mathrm{Fe}^{3+}(12 k)$. 

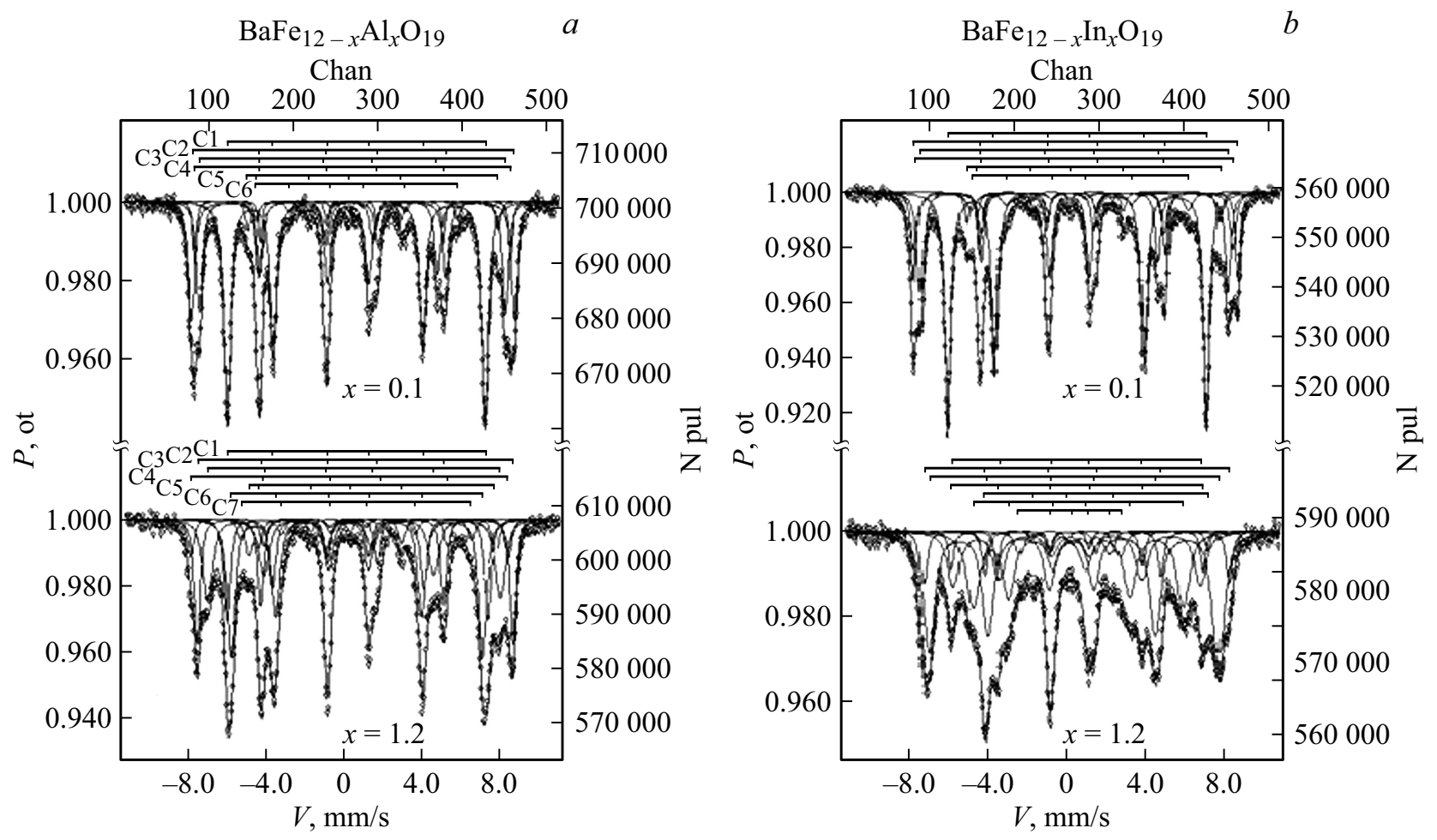

Рис. 3. Данные мессбауэровской спектроскопии для $\mathrm{BaFe}_{12-x} \mathrm{Al}_{x} \mathrm{O}_{19}(a)$ и $\mathrm{BaFe}_{12-x} \operatorname{In}_{x} \mathrm{O}_{19}(b)$, где $x=0.1$ и 1.2 .

Эффективные магнитные поля на ядрах $\mathrm{Fe}^{57}$ для $\mathrm{BaFe}_{10.8} \mathrm{Al}_{1.2} \mathrm{O}_{19}$ имеют следующий порядок: $\mathrm{B}_{\mathrm{eff}} \mathrm{Fe}(2 a)>$ $>\mathrm{B}_{\text {eff }} \mathrm{Fe}\left(4 f_{2}\right)>\mathrm{B}_{\text {eff }} \mathrm{Fe}\left(4 f_{1}\right)>\mathrm{B}_{\text {eff }} \mathrm{Fe}(12 k)>\mathrm{B}_{\text {eff }} \mathrm{Fe}\left(12 k^{1}\right)>$ $>\mathrm{B}_{\mathrm{eff}} \mathrm{Fe}(2 b)>\mathrm{B}_{\mathrm{eff}} \mathrm{Fe}\left(12 k^{2}\right)$. $\mathrm{B}$ то время, как для In-замещенного образца $(x=1.2)$ мы считаем, что диамагнитные ионы предпочтительно занимают $2 b$ и $2 a$-положения и ослабляют порядок обменных взаимодействий в связях $\mathrm{Fe}^{3+}(2 b)-\mathrm{O}-\mathrm{Fe}^{3+}(12 k)$ и $\mathrm{Fe}^{3+}(2 b)-\mathrm{O}-\mathrm{Fe}^{3+}(2 a)$. Поля на ядрах $\mathrm{Fe}^{57}$ для $\mathrm{BaFe}_{10.8} \mathrm{In}_{1.2} \mathrm{O}_{19}$ располагаются в следующем порядке: $\mathrm{B}_{\text {eff }} \mathrm{Fe}\left(4 f_{2}\right)>\mathrm{B}_{\text {eff }} \mathrm{Fe}\left(4 f_{1}\right)>\mathrm{B}_{\text {eff }} \mathrm{Fe}(2 a)>\mathrm{B}_{\text {eff }} \mathrm{Fe}(12 k)>$ $>\mathrm{B}_{\text {eff }} \mathrm{Fe}(2 b)>\mathrm{B}_{\text {eff }} \mathrm{Fe}\left(12 k^{1}\right)>\mathrm{B}_{\text {eff }} \mathrm{Fe}\left(12 k^{2}\right)$.

На рис. 4 представлены теоретические данные, которые были рассчитаны в соответствии с формулой (2). Для вычислений поля анизотропии были использованы данные для сферической формы кристаллитов в приближении низкой дисперсии по размерам. Данные рис. 5 подтверждают утверждение о различном влиянии ионов с разным электронным строением на электрофизические свойства (смещение резонансной частоты ЕФМР). Из рисунка можно отметить, что увеличение резонансной частоты ЕФМР (за счет увеличения магнитокристаллической анизотропии - МКА) характерно только для случая с замещением ионами $\mathrm{Al}^{3+}$. Остальные же ионы индуцируют снижение резонансной частоты $\left(f_{\text {res }}\right)$. Значение МКА для незамещенного гексаферрита бария $\mathrm{BaFe}_{12} \mathrm{O}_{19}$ составляет $16-17 \mathrm{kOe}$. Пик резонансной частоты отмечен в области 49-50 GHz. Замещение ионами алюминия индуцирует увеличение МКА для $\mathrm{BaFe}_{12-x} \mathrm{Al}_{x} \mathrm{O}_{19}$ с
$16-17 \mathrm{kOe}(x=0)$ до $23 \mathrm{kOe}(x=1.2)$. Это может быть объяснено ослаблением межрешеточного обменного взаимодействия $\mathrm{Fe}^{3+}-\mathrm{O}^{2-}-\mathrm{Fe}^{3+}$ за счет фрустрации магнитной структуры. В соответствии с данными мессбауэровской спектроскопии ослабление предпочтительно будет отмечено для взаимодействия позиций $2 b, 2 a$ и $12 k$ (ослабление дальнего порядка обменных взаимодействий связи $\left.\mathrm{Fe}^{3+}(2 b)-\mathrm{O}-\mathrm{Fe}^{3+}(12 k)\right)$. В зависимости от концентрации ионов алюминия будет наблюдаться конкуренция ослабления связей между $\mathrm{Fe}^{3+}(2 b)-\mathrm{O}-\mathrm{Fe}^{3+}(2 a)$ (для $x \leq 0.6$ ) и

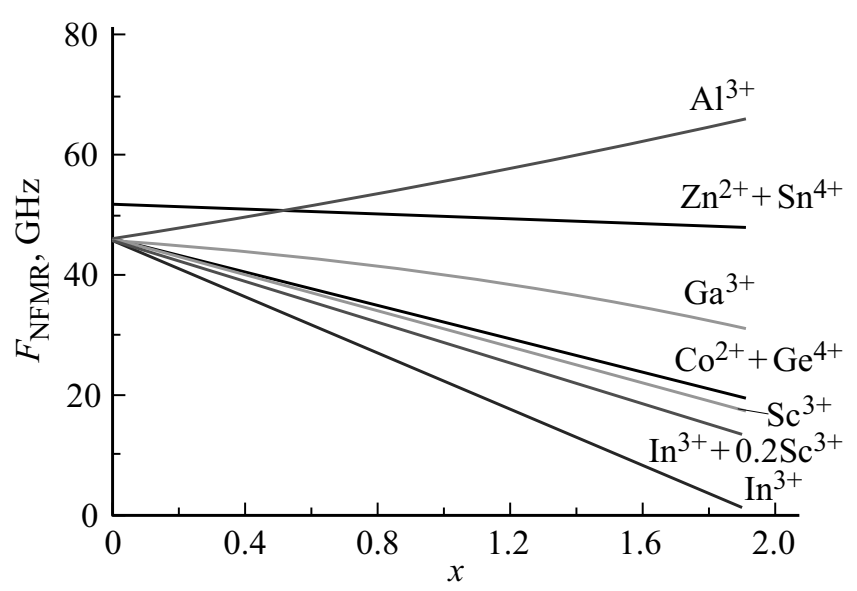

Рис. 4. Теоретические рассчитанные зависимости частоты ЕФМР для гексаферритов бария с замещением различными ионами. 
$a$

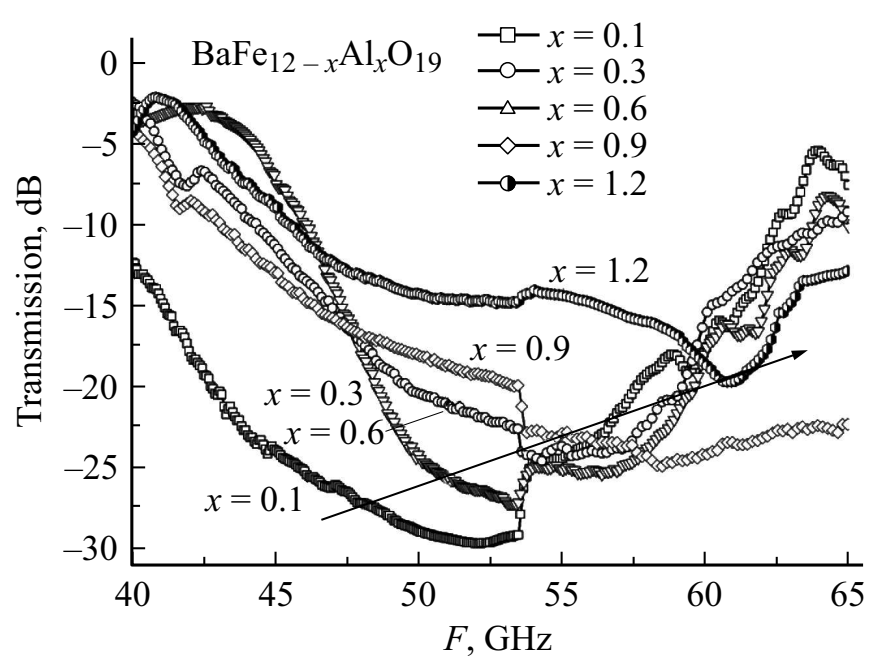

$b$

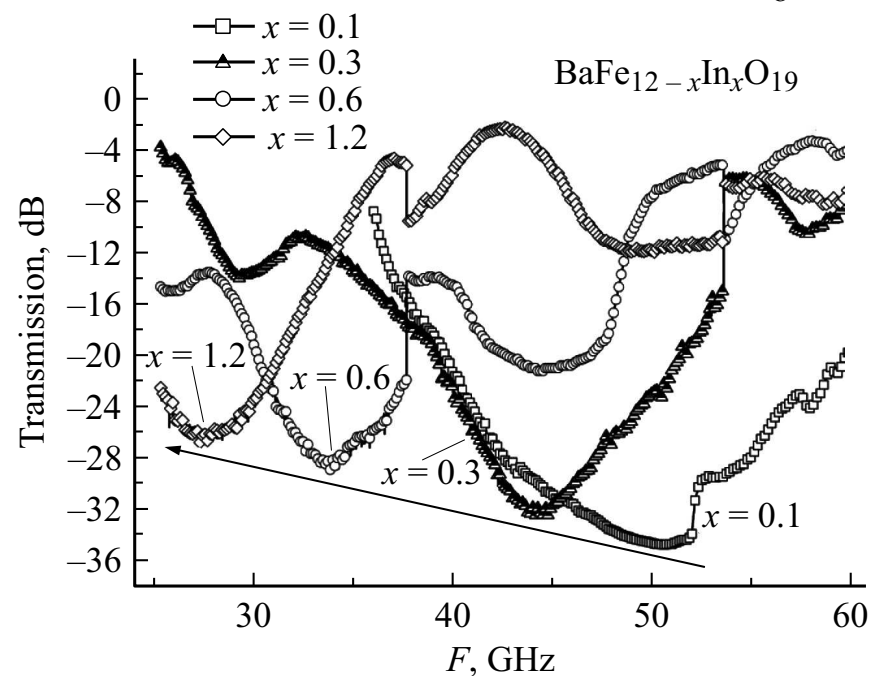

Рис. 5. Частотные зависимости коэффициента пропускания для образцов с различной концентрацией катионов $\mathrm{Al}^{3+}(a)$ и $\mathrm{In}^{3+}(b)$, где $0.1 \leq x \leq 1.2$.

$\mathrm{Fe}^{3+}(2 a)-\mathrm{O}-\mathrm{Fe}^{3+}(12 k)$ (для $\left.0.6<x \leq 1.2\right)$. Замещение ионами $\mathrm{In}^{3+}$ приводит к снижения величины МКА для $\mathrm{BaFe}_{12-x} \mathrm{In}_{x} \mathrm{O}_{19}$ с $16-17 \mathrm{kOe}(x=0)$ до $10-11 \mathrm{kOe}(x=1.2)$, что также может быть объяснено ослаблением межрешеточного обменного взаимодействия $\mathrm{Fe}^{3+}-\mathrm{O}^{2-}-\mathrm{Fe}^{3+}$ за счет фрустрации магнитной структуры. Но особенности межподрешеточного обменного взаимодействия для объяснения микроволновых характеристик должны быть проанализированы с позиции анализа электронного строения диамагнитных ионов и влияния энергии кристаллического поля.

На рис. 5 показаны спектры передачи (пропускания) ЭМИ через образцы с различной концентрацией катионов $\mathrm{Al}^{3+}$ (рис. $\left.5, a\right)$ и $\operatorname{In}^{3+}$ (рис. $5, b$ ). На рис. 6 представлены концентрационные зависимости амплитудночастотных характеристик электромагнитных свойств для образцов гексаферрита бария с замещением ионами $\mathrm{Al}^{3+}$ (рис. 6, $a$ ) и $\mathrm{In}^{3+}$ (рис. 6, $b$ ).

В диапазоне частот $20-65 \mathrm{GHz}$ для всех образцов наблюдается наиболее интенсивное снижение передачи из-за процессов поглощения (вызванное явлением ЕФМР). Значение абсцисс глобального минимума спектра передачи ЭМИ определяет резонансную частоту $\left(f_{\text {res }}\right)$. Значение ординаты глобального минимума спектра передачи ЭМИ определяет резонансную амплитуду ( $\left.A_{\text {res }}\right)$ процесса поглощения.

Для $\mathrm{Al}$-замещенных образцов $\mathrm{BaFe}_{12-x} \mathrm{D}_{x} \mathrm{O}_{19}$ было отмечено увеличение $f_{\text {res }}$ при увеличении концентрации ионов $\mathrm{Al}^{3+}$. Пик резонансной частоты $\left(f_{\text {res }}\right)$ сдвигался от $51 \mathrm{GHz}$ до $61 \mathrm{GHz}$ в диапазоне концентраций от $x=0.1$ до $x=1.2$ соответственно (рис. $6, b$ ). Результаты были в хорошем соответствии с теоретическими данными, полученными при численном расчете с использованием формулы (2).
Таким образом, увеличение концентрации ионов $\mathrm{Al}^{3+}$ приводит к уменьшению амплитуды $\left(A_{\text {res }}\right)$ с $-29.5 \mathrm{~dB}$ до $-20 \mathrm{~dB}$ (рис. 6,a). Для In-замещенных образцов $\mathrm{BaFe}_{12-x} \mathrm{D}_{x} \mathrm{O}_{19}$ было отмечено уменьшение $f_{\text {res }}$ при увеличении концентрации ионов $\mathrm{In}^{3+}$. Пик резонансной частоты сдвигался с $50.5 \mathrm{GHz}$ до $27 \mathrm{GHz}$ в диапазоне концентраций от $x=0.1$ до $x=1.2$ соответственно (рис. $6, d)$. Результаты эксперимента также были в хорошем соотвтетствии с теоретическим расчетом. Некоторое отклонение от линейной зависимости на частотных зависимостях для концентрации $x \geq 0.9$ может быть связано с внутренними микронапряжением (микродеформации в кристаллитах из-за большого ионного радиуса $\left.\mathrm{In}^{3+}\right)$. Амплитуда $A_{\text {res }}$ уменьшалась с $-34.9 \mathrm{~dB}$ до $-26.9 \mathrm{~dB}$ в том же диапазоне концентраций соответственно (рис. 6, $c$ ).

Влияние внешнего магнитного поля на амплитудночастотные характеристики исследуемых образцов отражает рис. 7.

Выше было отмечено, что наложение энергии внешнего магнитного поля способно приводить к увеличению МКА и соответственно смещать частоту ЕФМР в область больших частот. При исследовании образцов твердых растворов $\mathrm{BaFe}_{12-x} \mathrm{Al}_{x} \mathrm{O}_{19}$ (рис. 7, $a$ ) и $\mathrm{BaFe}_{12-x} \mathrm{In}_{x} \mathrm{O}_{19}$ (рис. 7, b) показано практически линейное увеличение МКА и как следствие линейное смещение резонансной частоты $\left(f_{\text {res }}\right)$ в область более высоких частот. Энергия внешнего магнитного поля увеличивает интенсивность внутриподрешеточных обменных взаимодействий, что увеличивает резонансную частоту спиновой прецессии. Возможность контролируемого управления амплитудно-частотными характеристиками посредством не только изменения химического состава, но и внешними магнитными полями открывает 

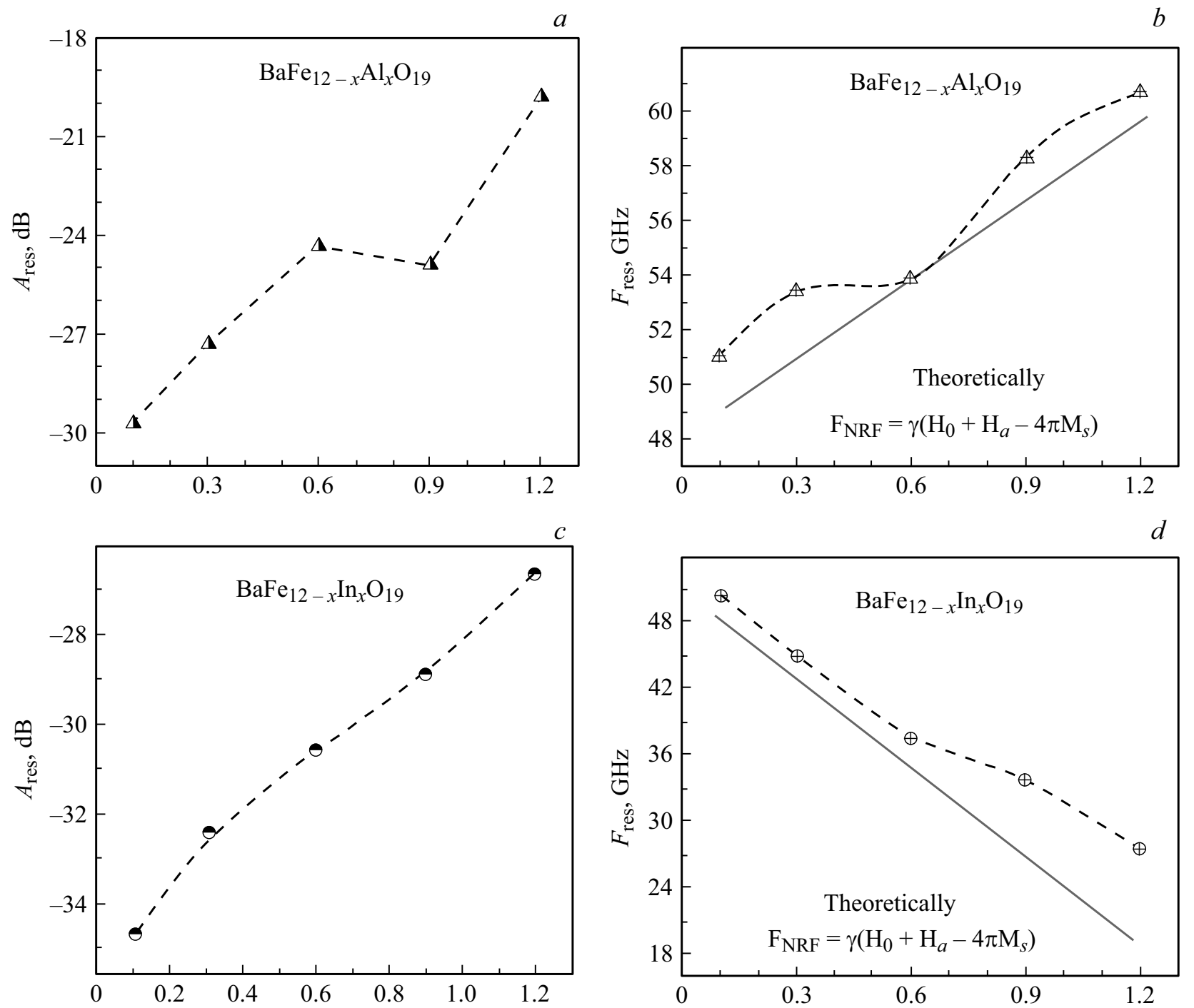

Рис. 6. Концентрационные зависимости резонансной амплитуды $\left(A_{\text {res }}\right)$ и резонансной частоты $\left(f_{\text {res }}\right)$ для образцов твердых растворов гексаферрита бария с замещением ионами $\mathrm{Al}^{3+}(a, b)$ и $\operatorname{In}^{3+}(c, d)$ в диапазоне концентраций $0.1 \leq x \leq 1.2$.
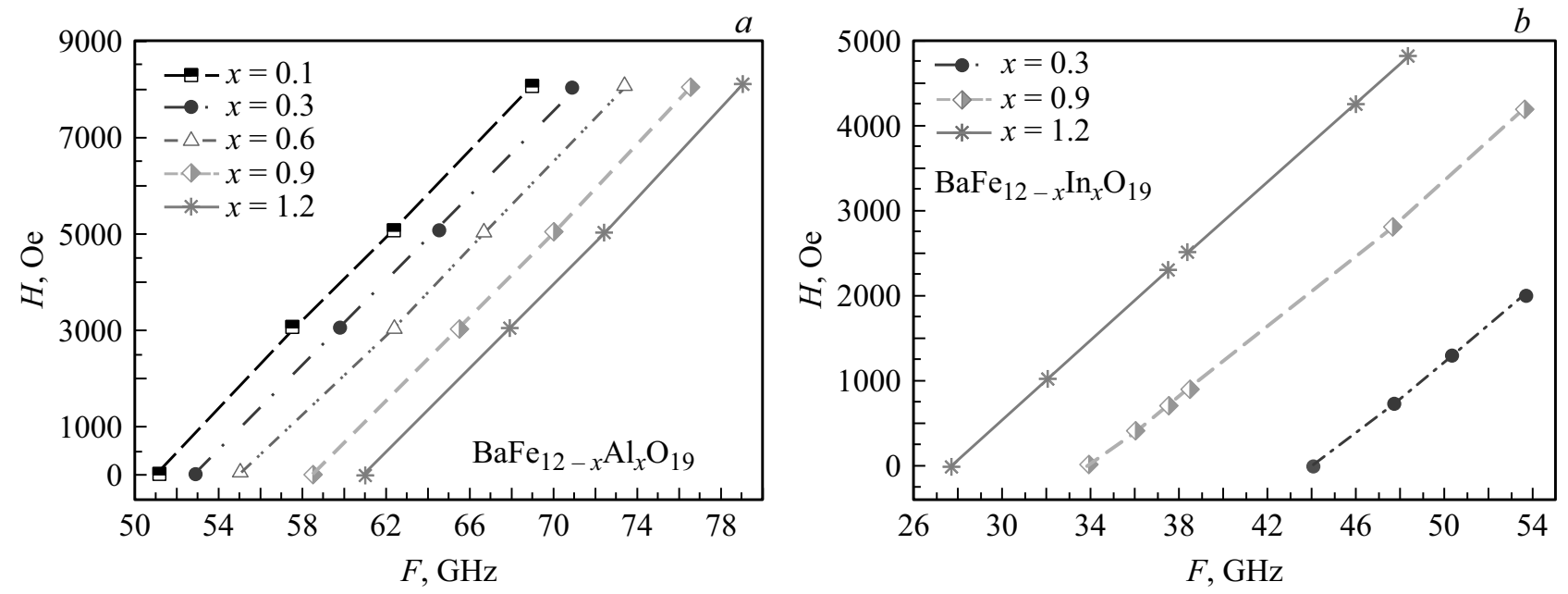

Рис. 7. Зависимость резнансной частоты ЕФМР от внешнего магнитного поля для образцов твердых растворов гексаферрита бария с замещением ионами $\mathrm{Al}^{3+}(a)$ и $\mathrm{In}^{3+}(b)$ в диапазоне концентраций $0.1<x<1.2$. 
серьезные перспективы для использования на практике данных материалов.

При поиске материалов, которые эффективно работают в микроволновом диапазоне, наибольший интерес представляет область, вызванная ЕФМР. Ферромагнитный резонанс приводит к потерям энергии электромагнитного поля, которые являются результатом ряда процессов при прецессии спинов катионов для ферро- или ферримагнетиков, связанных с дополнительными флуктуациями узлов кристаллической решетки. Прецессия спина при ЕФМР происходит под влиянием внутренних локальных магнитных полей в результате собственной магнитной анизотропии. Вектор намагниченности прецессирует вокруг оси легкого намагничивания. Физические принципы, ответственные за потери ЕФМР, те же, что и при индуцированном ферромагнитном резонансе. Это дополнительные флуктуации участков кристаллической решетки гексаферрита под влиянием спиновых волн. Взаимодействие спиновых волн с кристаллической решеткой приводит к тому, что часть энергии внешнего поля, возбуждающая тепловую спиновую прецессию и, следовательно, спиновые волны, превращается в тепловые флуктуации решетки. При этом частота ЕФМР определяется как энергией вращения вектора намагниченности в плоскости легкого намагничивания, так и энергией вращения из этой плоскости.

\section{4. Заключение}

Основная цель работы заключалась в проведении исследований корреляции химического состава, структуры и микроволновых характеристик твердых растворов гексаферрита бария $\mathrm{BaFe}_{12-x} \mathrm{D}_{x} \mathrm{O}_{19}(0.1 \leq x \leq 1.2)$ с замещением диамагнитными ионами $\mathrm{Al}^{3+}$ и $\mathrm{In}^{3+}$. Это потребовало прецизионных исследований методами порошковой дифракции, мессбауэровской спектроскопии и высокочастотных измерений коэффициентов пропускания. Методом порошковой дифракции было показано, что зависимость параметров элементарной ячейки от концентрации тесно связана с эффективным ионным радиусом ионов-заместителей $\left(\mathrm{Al}^{3+}-0.540 \AA\right.$ и $\mathrm{In}^{3+}-$ $0.940 \AA)$. Характер распределения диамагнитных ионов определялся методом мессбауэровской спектроскопии. Показано, что распределение диамагнитных ионов в малых концентрациях $(x<0.3)$ носят схожий характер для Al- и In-замещенных составов. При увеличении концентрации ионов-заместителей характер распределения отличен для Al-замещенных и In-замещенных образцов. Это обстоятельство по-разному отражается на ослаблении межподрешеточных обменных взаимодействий в цепочках $\mathrm{Fe}^{3+}-\mathrm{O}^{2-}-\mathrm{Fe}^{3+}$ за счет различных механизмов фрустрации магнитной структуры. Показано, что уровень концентрационного замещения и природа иона-заместителя по-разному влияют на амплитудночастотные характеристики твердых растворов гексаферрита бария $\mathrm{BaFe}_{12-x} \mathrm{D}_{x} \mathrm{O}_{19}(0.1 \leq x \leq 1.2) \mathrm{c} \mathrm{Al}^{3+}$ и $\mathrm{In}^{3+}$.
Потери энергии ЭМИ при взаимодействии с исследуемыми образцами обусловлены процессами поглощения за счет естественного ферромагнитного резонанса. В основе данных процессов лежит изменение магнитокристаллической анизотропии при изменении химического состава. Для Al-замещенных составов отмечено практически линейное увеличение резонансной частоты $\left(f_{\text {res }}\right)$ при снижении резонансной амплитуда $\left(A_{\text {res }}\right)$. Для In-замещенных образцов, напротив, отмечено резкое снижение резонансной частоты, что обусловлено снижением магнитокристаллической анизотропии при фрустрации магнитной структуры. Дополнительный вклад в поле анизотропии может быть обусловлен возникновением внутренних микронапряжений и микродеформаций. Изменение анизотропии было объяснено ослаблением интенсивности межподрешеточных сверхобменных взаимодействий. Было показано, что энергия внешнего магнитного поля практически линейно смещает резонансную частоту в область более высоких частот, что объясняется дополнительным энергетическим вкладом в рост МКА.

Результаты исследований микроволновых характеристик в частотном диапазоне $20-65 \mathrm{GHz}$ представляют большой интерес с практической точки зрения за счет возможности контролируемого управления электродинамическими характеристиками как путем изменения химического состава гексагонального феррита, так и воздействием внешнего магнитного поля.

Авторы выражают глубокую благодарность А.М. Балагурову за помощь в интерпретации экспериментальных данных.

\section{Список литературы}

[1] V. Adelskold. Avk. Miner. A 1, 12 (1938).

[2] S.V. Trukhanov, A.V. Trukhanov, V.G. Kostishyn, L.V. Panina. Dalton Transactions 46, 9010 (2017).

[3] O.P. Aleshko-Ozhevskii, R.A. Sizov, I.I. Yamzin, V.A. Lubimtsev. JETP 28, 425 (1969).

[4] L. Wang, H. Yu, X. Ren, G. Xu. J. Alloys Compd. 588, 212 (2014).

[5] P. Meng, K. Xiong, L. Wang, S. Li, Y. Cheng, G. Xu. J. Alloys Compd. 628, 75 (2015).

[6] V.A. Turchenko, A.V. Trukhanov, I.A. Bobrikov, S.V. Trukhanov, A.M. Balagurov. Crystallogr. Rep. 60, 629 (2015).

[7] A.V. Trukhanov, L.V. Panina, S.V. Trukhanov, V.A. Turchenko, M. Salem. Chin. Phys., B 25, 016102-6 (2016).

[8] D.A. Vinnik, D.A. Zherebtsov, L.S. Mashkovtseva, S. Nemrava, M. Bischoff, N.S. Perov, A.S. Semisalova, I.V. Krivtsov, L.I. Isaenko, G.G. Mikhailov, R. Niewa. J. Alloys Compd. 615, 1043 (2014).

[9] A.M.Y. El-Lawindy, S.A. Mansour, M. Hafiz, H.H. Hassan, A.A. Ali. Int. J. Appl. Ceram. Technol. 7, 868 (2010).

[10] X. Liu, J. Wang, L.M. Gan, S.C. Ng, J. Ding. J. Magn. Magn. Mater. 184, 344 (1998).

[11] E. Richter, T.J.E. Miller, T.W. Neumann, T.L. Hudson. IEEE Trans. Industry Appl. 1A-21, 644 (1985). 
[12] Q.A. Pankhurst, R.S. Pollard. J. Phys.: Condens. Mater. 5, 8487 (1993).

[13] Y. Tokunaga, Y. Kaneko, D. Okuyama, S. Ishiwata, T. Arima, S. Wakimoto, K. Kakurai, Y. Taguchi, Y. Tokura. Phys. Rev. Lett. 105, 257201-4 (2010).

[14] B. Warcholinski, A. Gilewicz, T.A. Kuznetsova, T.I. Zubar, S.A. Chizhik, S.O. Abetkovskaia, V.A. Lapitskaya. Surf. Coatings Technol. 117, 319 (2017).

[15] E.V. Sadyrin, B.I. Mitrin, S.M. Aizikovich, T.I. Zubar. Mater. Phys. Mech. 28, 1 (2016).

[16] G. Tan, X. Chen. J. Magn. Magn. Mater. 327, 87 (2013).

[17] A.V. Trukhanov, V.O. Turchenko, I.A. Bobrikov, S.V. Trukhanov, I.S. Kazakevich, A.M. Balagurov. J. Magn. Magn. Mater. 253, 393 (2015).

[18] A.V. Trukhanov, S.V. Trukhanov, L.V. Panina, V.G. Kostishyn, D.N. Chitanov, I.S. Kazakevich, An.V. Trukhanov, V.A. Turchenko, M. Salem. Ceram. Int. 43, 5635 (2017).

[19] S.V. Trukhanov, A.V. Trukhanov, V.G. Kostishin, L.V. Panina, I.S. Kazakevich, V.A. Turchenko, V.V. Kochervinskiy. JETP Lett. 103, 100 (2016).

[20] A.V. Trukhanov, S.V. Trukhanov, V.G. Kostishin, L.V. Panina, M.M. Salem, I.S. Kazakevich, V.A. Turchenko, V.V. Kochervinskii, D.A. Krivchenya. Phys. Solid State 59, 4, 737, (2017)

[21] L. Li, K. Chen, H. Liu, G. Tong, H. Qian, B. Hao. J. Alloys Compd. 11, 557 (2013).

[22] N. Velhal, G. Kulkarni, D. Mahadik, P. Chowdhury, H. Barshilia, V. Puri. J. Alloys Compd., 682, 730 (2016).

[23] L.I. Krenev, E.V. Sadyrin, S.M. Aizikovich, T.I. Zubar. Springer Proc. Phys. 193, 184 (2017).

[24] B. Warcholinski, A. Gilewicz, O. Lupicka, A.S. Kuprin, G.N. Tolmachova, V.D. Ovcharenko, I.V. Kolodiy, M. Sawczak, A.E. Kochmanska, P. Kochmanski, T.A. Kuznetsova, T.I. Zubar, A.L. Khudoley, S.A. Chizhik. Surf. Coatings Technol. 920, 309 (2016).

[25] F.A. Miranda, F.W. Van Keuls, R.R. Romanofsky. IEEE Trans. Microwave Theory Tech. 48, 1181 (2000).

[26] J.-S. Kim, Y.-H. Lee, B. Lee, J.-C. Lee, J.J. Choi, J.Y. Kim. J. Electrical Eng. Technology 9, 1, 273, (2014).

[27] L. Vovchenko, L. Matzui, O. Brusylovets, V. Oliynyk, V. Launets, A. Shames, O. Yakovenko, N. Skoryk. Mat.-wiss. u. Werkstofftech 47, 2-3, 139 (2016).

[28] D. Chen, Y. Liu, Y. Li, K. Yang, H. Zhang. J. Magn. Magn. Mater. 37-38, 65 (2013)

[29] T. Kuznetsova, T. Zubar, S. Chizhik, A. Gilewicz, O. Lupicka, B. Warcholinski. J. Mater. Eng. Perform. 25, 5450 (2016).

[30] V. Zavaleyev, J. Walkowicz, T. Kuznetsova, T. Zubar. Thin Solid Films 153, 638 (2017).

[31] D.A. Vinnik, A.B. Ustinov, D.A. Zherebtsov, V.V. Vitko, S.A. Gudkova, I. Zakharchuk, E. Lähderanta, R. Niewa. Ceram. Int. 41, 12728 (2015).

[32] S.V. Trukhanov, A.V. Trukhanov, V.O. Turchenko, V.G. Kostishin, L.V. Panina, I.S. Kazakevich, A.M. Balagurov. J. Magn. Magn. Mater. 417, 130 (2016).

[33] S.V. Trukhanov, A.V. Trukhanov, V.G. Kostishyn, L.V. Panina, An.V. Trukhanov, V.A. Turchenko, D.I. Tishkevich, E.L. Trukhanova, V.V. Oleynik, O.S. Yakovenko, L.Yu. Matzui, D.A. Vinnik. J. Magn. Magn. Mater. 442, 300 (2017).

[34] T.A. Kuznetsova, T.I. Zubar, V.A. Lapitskaya, K.A. Sudzilouskaya, S.A. Chizhik, A.L. Didenko, V.M. Svetlichnyi, M.E. Vylegzhanina, V.V. Kudryavtsev, T.E. Sukhanova. IOP Conf. Ser. Mater. Sci. Eng. 256, 1 (2017).
[35] S. Kuprin, T.A. Kuznetsova, A. Gilewicz, G.N. Tolmachova, V.D. Ovcharenko, S.O. Abetkovskaia, T.I. Zubar, A.L. Khudoley, S.A. Chizhik, O. Lupicka, B. Warcholinski. Probl. At. Sci. Technol. 6106, 147 (2016).

[36] A.V. Trukhanov, S.V. Trukhanov, L.V. Panina, V.G. Kostishyn, I.S. Kazakevich, An.V. Trukhanov, E.L. Trukhanova, V.O. Natarov, V.A. Turchenko, M.M. Salem, A.M. Balagurov. J. Magn. Magn. Mater. 426, 487 (2017).

[37] S.V. Trukhanov, A.V. Trukhanov, V.A. Turchenko, An.V. Trukhanov, E.L. Trukhanova, D.I. Tishkevich, V.M. Ivanov, T.I. Zubar, M. Salem, V.G. Kostishyn, L.V. Panina, D.A. Vinnik, S.A. Gudkova. Ceram. Int. 44, 290 (2018).

[38] H.M. Rietveld. J. Appl. Cryst. 2, 65 (1969).

[39] http://www.ill.eu/sites/fullprof/

Редактор Т.Н. Василевская 